\title{
Automatic Face Recognition Using Gabor Wavelet and Robust Structured Spares Representation
}

\author{
R.Madhura ${ }^{1}$, P.Suthanthira Devi ${ }^{2}$ \\ ${ }^{I} P G$ Student, Department of Computer Science \& Engineering, AIHT, Chennai \\ ${ }^{2}$ Assistant Professor, Department of Computer Science \& Engineering, AIHT, Chennai
}

\begin{abstract}
Automatically facial recognition has become more and more important today. But FR still remains a challenge problem in computer vision .so in this paper we proposed a new approach that use Gabor wavelet for facial feature extraction and robust structured sparse representation for classification. Gabor wavelet has beenwidely used in the face recognition task because it's good imitation of human visual. The structured sparse representation based classification (SSRC) is used for classifying the test images robustly. Unlike sparse representation based classification (SRC), the SSRC explicitly takes structure of the dictionary into account for a better classification.
\end{abstract}

Keywords: Gabor Wavelet, Structured sparse representation classification,Face Recognition.

\section{Introduction}

Human face detection and recognition is one of an energetic area of study, straddling a number of disciplines such as pattern recognition, image processing and computer vision with wide range of applications such as identity verification, video-surveillance, facial expression extraction, and advanced human-computer interaction. A necessitate to develop robust face recognition algorithm is due to highly complex distribution and worse recognition performance as a result of wide-range variations of human face due to pose, illumination, and expression.

Face detection is a process how to determine the position,size and pose of a face image in the input picture. It is a keyproblem of automatic face recognition system and a keytechnology of face information processing. It has attractedsignificant attention over the past few years in the field ofcomputer vision and pattern recognition.

Face recognition is the process of identifying humanwith the use of face characteristics. Face recognition is agrowing research area in biometrics; particularly it has a hugeinvolvement in law enforcement, commercial, and socialservices.

From the last few decades, several feature extractionmethods have been proposed for face recognition and haveachieved high recognition rates. However, it is still difficultfor a machine to recognize human faces accurately with asingle training image due to low interpersonal variations andlarge intrapersonal variations, which happened due tovariations in pose, expression, illumination, scaling and other.

Wavelet transform is a good signal "space-frequency"analysis tool. It has good local properties whenever in thetime or the frequency Domain. As wavelet graduallyrefined high-frequency signals in the time domain orfrequency domain, it can focus the signal on any details.

Two-dimensional wavelets transform is realized bycomputing the convolutions of a bank of twodimensionalGabor filters and the grey values of pixels in an area arounda given position in an image. Twodimensional Gaborwavelets transform seems to be a good approximation to thereceptive fields of the simple cells in the visual cortex ofmammalians and it's the compromise accuracy in the timedomain and frequency domain. So, two-dimensional Gabor wavelets have been introduced into the face recognition area.

Face detection and recognition have many methods today, the Gabor wavelet function has feature of space locality, frequency selectivity and direction selectivity. It can detect and extract frequency and direction information of facial area. It is an effective tool in facial image analysis and description. It has a robust facial feature description.

Based on this characteristic, the feature extraction is addressed by using Gabor wavelets and classification by structured sparse on face images. 


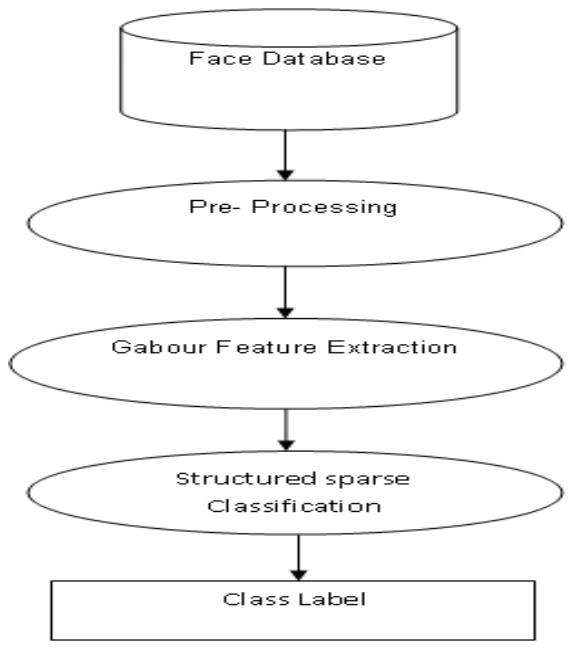

Fig.1 Generic Structure of Face Recognition

Firstly, it utilizes Gabor wavelet toextract face feature and constructs human face training samples set. Then, it uses structured sparse for classificationmachine to determine whether existing human face or not inthe input picture and the human face position.

\section{Related Works}

Robust Sparse Coding for Face Recognition: This paper proposed a new scheme, namely the robust sparse coding (RSC), by modeling the sparse coding as sparsity constrained robust regression problem. The RSC seeks for the MLE (maximum likelihood estimation) solution of the sparse coding problem, and it is much more robust to outliers (e.g., occlusions, corruptions, etc.) than SRC. An efficient iteratively reweighted sparse coding algorithm is proposed to solve the RSC model.

Robust Face Recognition via Sparse Representation: Based on a sparse representationcomputed by '1minimization, they propose a general classification algorithm for image-based recognition. This new framework provides new insights in to two crucial issues in face recognition: feature extraction and robustness to occlusion. Face Recognition Based on Gabor with 2DPCA and PCA: Unlike a sine wave, a wavelet has itsenergy concentrated in time. Wavelets are well suited forthe analysis of transient, time-varying signals. When processing an image uses a group Gabor nuclear with five scale and eightdirections, the data obtained is enormous.

Face Recognition based on Sparse Representation and Error Correction SVM:SparseRepresentation Discrimination Analysis (SRDA) by combiningthe sparse representation theory and the manifold learning modeltogether. The SRDA algorithm can maintain not only the sparsereconstruction relationship of original data, but also the spatialstructure in low dimensional space. Then, the SRDA feature isintegrated with the error correction SVM to build a new facerecognition system.

Face Detection Using Gabor Wavelet and SVM:Firstly, the Gaborwavelet features of human face and nonhuman face samplesset are extracted and the training samples set are constructed.Then, support vector machine are trained and the optimalclassification decision function is calculated. The comparativeexperiment results show that the method has thecharacteristics such as simplification, lower calculationcomplexity, high efficiency, better detection and allocationaccuracy for facial and vertical human face image.

\section{III.Feature extraction via Gabor wavelet}

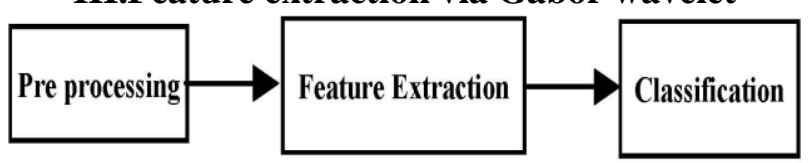

Fig 2.Block diagram of a typical face recognition system

In face recognition system the feature based method finds the important features on the face and represents them in an efficient way. Physiological studies found thatthe cells in the human virtual cortex can be selectivelytuned to orientation and to spatial frequency. This confirmation that the response of the simple cell could be approximated using Gabor Wavelet. 
In 1946, Gabor proposed one dimension Gabor wavelet.In 1985, Daugman J proposed two dimensions Gabor wavelet.Gabor wavelet is used in the field of human face recognitionfor its correlation with biologic. The receptive field spacestructure of the mammalian visual simple cortical cell can bedescribed with Gabor mathematic function. The simplecells are irresponsive to large acreage diffused light and havestrictly selectivity to the edge position and azimuth. Gaborwavelet feature description method is a integrated methodwhich is based on gray and features. It has some greatproperties such as space position selection, directionselection, frequency selection and orthogonality. It extracts the Gabor wavelet coefficients of different direction andscale as features from data gray distribution. It uses a groupof feature and position to descript objects. Simultaneity, theGabor function has good space and frequency resolution. So,it is a good detector. The extraction of local features in animage can be effectively done using Gabor wavelets.

Wavelet-based analysis of signals is an interesting and relatively new tool.The 2-D Gabor filters is a linear transform used for edgedetection and texture discrimination. Frequency andorientation representations of Gabor filters are similar tothose of the human visual system, and they have beenfound to be very appropriate for texture representationand discrimination. The Gabor kernels show strongcharacteristics of spatial locality and orientationselectivity, which are suitable for deriving local anddiscriminating features for facial expressionclassification. The transform of Gabor wavelet can bedefined as:

$$
\begin{gathered}
\varphi(x, y, \omega, \theta)=\frac{1}{2 \pi \sigma^{2}} e^{-\left(\frac{x^{\prime 2}+y^{\prime 2}}{2 \sigma^{2}}\right)}\left[e^{t \bar{\omega} x^{\prime}}-e^{\frac{\bar{\omega} \sigma^{2}}{2}}\right] \\
x^{\prime}=x \cos \theta+y \cos \theta \\
y^{\prime}=-x \sin \theta+y \cos \theta
\end{gathered}
$$

In this equation, $(\mathrm{x}, y)$ is the pixel position in the spatialdomain $\omega$, is the radial center frequency, $\theta$ is theorientation of Gabor filter, and $\sigma$ is the standarddeviation of the round Gaussian function along the xandyaxes.

\section{Classification Using Structured Sparse}

Classification is one of the most fundamental problems in machine learning and has numerous applications in different areas including computer vision. Recently, there has been an increasing interest in classification problems where the data across multiple classes come from a collection of low-dimensional linear sub-space. The important problems in face recognition is, the data lie in multiple low-dimensional subspaces of a high dimensional ambient space. However, most existing classification methods do not explicitly take into account the multi-subspace structure of the data. An important class of methods that deals with data on multiple subspaces relies on the notion of sparsity.

Specifically, the sparse representation-based classification (SRC)method looks for the sparsest representation of a testexample in a dictionary composed of all training data acrossall classes.

When it comes to the problem of robust classification,the SRC method offers a great advantage over many classificationmethods since it can effectively deal with corrupteddata within the same sparse representation framework. While sparse representation-based methodshave been shown to be effective for classification, there stillremain questions about classification in the multi-subspacesetting using sparse representation which have not been sufficientlyexplored or have not been answered yet. When the number of training data in each class issmall, sparse recovery methods have good theoretical guarantees.However, classification algorithms do not performwell.

\subsection{Classification via structured sparseRepresentation.}

Although the SRC method described above has a goodperformance in reconstruction and classification, it doesnot explicitly take into account the multi-subspacestructure of the data. Thedictionary of the training data has a structure in whichdata from each class form few blocks of the dictionary.

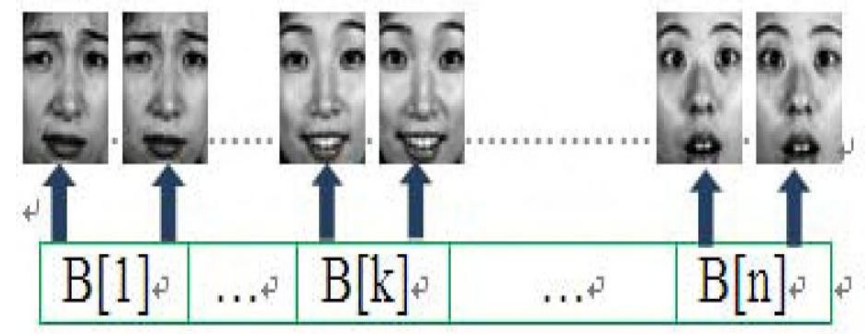

Fig 3. Block Structure of FR

The structured sparse representation is looking for therepresentation of the test sample that involves leastblocks from the dictionary. Formally the objection can be formulated as: 


$$
P_{l_{q / l_{o}}}: \min \sum_{i=1}^{n} I(|| C[i] \| \mid q>0)
$$

Where $I(\cdot)$ is an indicator function, $\mathrm{q} \geq 1\}$ and $c[i] \epsilon \mathrm{R}^{\mathrm{mi}}$ are the entries of $\mathrm{c}$ corresponding to the $\mathrm{i}$-th block of the dictionary, which contains $\mathrm{m}_{\mathrm{i}} \mathrm{values} . B[i] \in \mathrm{R}{ }^{\mathrm{DXmI}}$ are training sample with $D$ dimensions andorganized in blocks as shown in the figure above. Note theoptimization problem seeking for the minimum numberof nonzero coefficient blocks to represent the test sample, and $P l q / l o$ is an NP-hard problem. The $l_{i}$ relaxation of this problem is given as:

$$
P l_{q / l_{1}}: \min \sum_{i=1}^{n}\|C[i]\| q
$$

Where $q \geq 1 . P l q / l_{l}$ is a convex optimation problem which can be solved in polynomial time. The SRCobjection $P l_{l}$ can be thought as a structured sparserepresentation with the objection $P l_{l} / l_{l}$ By solving then $P l q / l_{l}$ problem, we reconstruct the test sample forcomparison, and the ith-class with the minimumreconstruction residual should be the class that the testimage belongs to.

\section{Conclusion}

In this paper, a new method for automatically facial recognition was proposed, which uses Gabor wavelet for feature extraction and SSRC forclassification. The experimental results have shown that the Gabor plus SSRC method has improved the recognition rate compared with some previous methods including SVM and SRC, especially when the test images facing different degree of corruption and occlusion.

\section{References:}

[1] D. Hoiem, A. N. Stein, A. A. Efros, and M. Hebert (2011), "Recovering occlusion boundaries from a single image" IEEE Int. ConfComput. pg no. 1-8.

[2] H. Jia and A. M. Martinez, (2010) "Face recognition with occlusions in the training and testing sets" IEEE Int. Conf. Autom. Face Gesture Recognit, pg no. 1-6.

[3] J. Wright, A. Yang, A. Ganesh, S. Sastry, and Y. Ma, (2009) "Robust face recognition via sparse representation", IEEE Trans. Pattern Anal. Mach Intell, pg no. 210-227.

[4] M. Yang, L. Zhang, J. Yang, and D. Zhang, (2011) "Robust sparse coding for face recognition", IEEE Int. Conf. Comput. Vis. Pattern Recognit, pg no. 625-632.

[5] Y. C. Eldar and M. Mishali, (2009)“Robust recovery of signals from a structured union of subspaces", IEEE Trans. Inf. Theory.

[6] Jing Wang and ChenganGuo,(2012) "Face Recognition based on Sparse Representation and Error Correction SVM", IEEE Trans. Conf Computational Intelligence, pg no. 10-15

[7] Zhao Lihong, Yang Caikun, Pan Feng and Wang Jiahe, (2012) "Face recoginition based on gabor with 2DPCP and PCA" 24th Chinese Control and Decision Conference no. 1-6.

[8] Zhang Guo-yun, Guo Long-yuan , Wu Jian-hui, Li Hong-min, Guo Guan-qi, (2010)“ Face Detection Using Gabor Wavelet and SVM", International Conference on Computer Application and System Modeling .

[9] M. Yang, L. Zhang, J. Yang, and D. Zhang, (2012) "A Face Recognition Algorithm Using Gabor Wavelet and Orthogonal Locality Preserving Projection", IEEE Trans on image processing.

[10] Y. C. Eldar, P. Kuppinger, and H. Bolcskei. Compressed sensing of block-sparse signals: Uncertainty relations and efficient recovery.IEEE Trans. Signal Processing, 58(6):3042-3054, June 2010. 